\title{
Resonances in the photodetachment cross section of $\mathrm{Au}_{2}^{-}$
}

\author{
G. F. Gantefor, D. M. Cox, and A. Kaldor \\ Corporate Research Laboratories, Exxon Research and Engineering Company, Annandale, \\ New Jersey 08801
}

\begin{abstract}
This paper reports the observation of autodetaching resonances in photoelectron spectroscopy of $\mathrm{Au}_{2}^{-}$according to the process $\mathrm{Au}_{2}^{-}+h v \Rightarrow\left(\mathrm{Au}_{2}^{-}\right)^{*} \Rightarrow \mathrm{Au}_{2}+e^{-}$. They appear as sharp features superimposed upon a nearly constant signal associated with direct photodetachment process from $\mathrm{Au}_{2}^{-}: \mathrm{Au}_{2}^{-}+h v \Rightarrow \mathrm{Au}_{2}+e^{-}$. By altering the cluster expansion conditions, the dimer anion temperature can be varied. This leads to changes in the linewidth and relative intensities of the resonances and allows ground state and hot band transitions to be identified. The resonances correspond to electronic transitions to a long-lived excited state of $\mathrm{Au}_{2}{ }^{-}$with a significantly lower vibrational frequency $\left(128 \pm 2 \mathrm{~cm}^{-1}\right)$ than that of the neutral ground state $\left(190.9 \mathrm{~cm}^{-1}\right)$, or even the anion ground state $\left(149 \mathrm{~cm}^{-1}\right)$. It decays via electronic autodetachment in a two-electron process similar to autoionization of neutral atoms.
\end{abstract}

\section{INTRODUCTION}

During the last decade, spectroscopy of negative ions ${ }^{1-3}$ has become increasingly important in the study of the electronic and geometric structures of negative and neutral atoms, molecules, and clusters. Until the 1980's, most spectroscopic information on anions was obtained by electron scattering

$$
\begin{aligned}
e^{-}+M \Rightarrow\left(M^{-}\right)^{*} & \Rightarrow M+e^{-} \\
& \Rightarrow M^{*}+e^{-} \Rightarrow \cdots
\end{aligned}
$$

observing various decay channels. ${ }^{4.5}$ Excited states of anions in the continuum manifest themselves as resonances in the electron scattering cross section. A manifold of resonances is found for most atoms and even simple molecules. ${ }^{4}$

Availability of intense anion sources ${ }^{1,2,6}$ now allows such "temporary negative ions" to be created directly using tunable radiation

$$
\begin{aligned}
h v+M^{-} \Rightarrow\left(M^{-}\right)^{*} & \Rightarrow M+e^{-} \\
& \Rightarrow M^{*}+e^{-} \Rightarrow \cdots .
\end{aligned}
$$

This method is very similar to autoionization studies of neutral atoms and molecules which has proven to be a powerful tool for the determination of the structural and dynamical properties of atoms and molecules. ${ }^{7}$ The high resolution of the laser radiation used for photodetachement allows observation of vibrational and rotational fine structure and determinations of lifetimes. Resonances have been observed in larger molecules, where the excited electron is bound by a permanent dipole moment of the neutral core. ${ }^{8,9}$ Recently, Smalley's group reported resonances ${ }^{10}$ with a striking vibrational structure for $\mathrm{Au}_{6}^{-}$, which has no permanent dipole moment. The excited state of the anion is interpreted as a weakly bound image charge state similar to surface states known in solid state physics which decay via vibrational autodetachment. Because of the very low binding energy of the excited electron, the observed vibrational frequencies are believe to be similar to the ones of neutral ground state of $\mathrm{Au}_{6}$.

In this paper, we present results of studies of autodetaching resonances of $\mathrm{Au}_{2}^{-}$. For this much simpler system, the vibrational frequencies of the anion and the neutral are well known ${ }^{11,12}$ and thus the interpretation of the vibrational structure is easier. Our data suggest that $\mathrm{Au}_{2}^{-}$is undergoing a different type of autodetaching process, in which an excited electronic state of the anion decays by electronic autodetachment in a two-electron transition.

\section{EXPERIMENTAL}

The experimental setup is similar to that described in an earlier publication. ${ }^{11}$ In short, charged and neutral metal clusters are produced by a laser vaporization source and, entrained in an inert carrier gas, undergo a supersonic expansion. After passing through a skimmer, the anions are accelerated by a pulsed high voltage $(\sim 700 \mathrm{~V})$ and focused into the interaction zone of an electron collector. This separates the anions into bunches of defined mass due to their differing times-of-flight. The desired bunch is irradiated by a pulse of tunable laser light and the detached electrons are extracted by a constant electrical field and detected by a channeltron. For measurements of the relative photodetachement cross section, both the anion and the laser flux have to be kept as low as possible to avoid space charge effects, power broadening, and saturation. The typical light flux is about $1 \mu \mathrm{J} / \mathrm{cm}^{2}$, the photon energy resolution is better than 1 $\mathrm{cm}^{-1}$. Spectra are taken by recording the intensity of the detached electrons while scanning the wavelength of the dye laser. The electron intensity is normalized to the laser light flux. The large uncertainty of the relative intensities of resonances is mostly due to the instability of the anion intensity produced by the laser vaporization source. Spectra are averages of several scans, whereas each data point in a scan is an average of ten laser shots. The step width is $0.009 \mathrm{~nm}$.

\section{RESULTS}

Figure 1 shows a typical spectrum obtained from photodetachment of $\mathrm{Au}_{2}^{-}$taken at a relative high photon flux $\left(\sim 100 \mu \mathrm{J} / \mathrm{cm}^{-1}\right)$. In principle, all detached electrons irrespective of their kinetic energy (KE) should be collected with such a technique. In our particular geometry, the collection efficiency decreases slowly for electrons with $>50$ $\mathrm{meV} \mathrm{KE}$ since the fastest electrons can hit nearby surfaces 


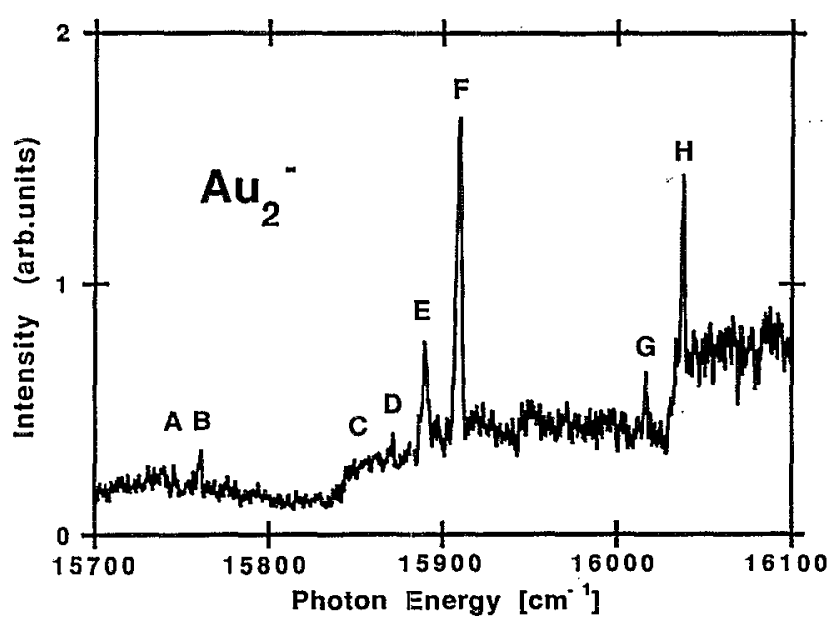

FIG. 1. Dependence of the $\mathrm{Au}_{2}{ }^{-}$photodetachment signal on the photon energy at a photon fiux of approximately $100 \mu \mathrm{J} / \mathrm{cm}^{2}$. At this relative high flux, the observed resonances are strongly suppressed and power broadened. The electrons are extracted by a small bias voltage of $2 \mathrm{~V}$ and due to steradian effects, electrons with kinetic energy higher than $\sim 50 \mathrm{meV}$ are detected with a lower probability. The low and fairly smooth underlying signal corresponds to the direct photodetachment process $\mathrm{Au}_{2}^{-}$ $=h \nu \Rightarrow \mathrm{Au}_{2}+e^{--}$, while the resonances (peaks $A-H$ ) are transitions to an excited state of the anion which subsequently autodetaches. The vibrational temperature of the anion is known from other measurements to be $165 \pm 30$ $\mathrm{K}$ (Ref. 11). The positions of features $A$ and $C$ are also marked. These two features are not observable at this high photon flux, but are clearly visible at $1 \mu \mathrm{J} / \mathrm{cm}^{2}$ (see Fig. 2).

and go undetected. In the absence of any long-lived excited negative ion state, an energetically accessible channel for direct detachement simply leads to a step increase in the total electron signal, i.e., the two signal increases near 15840 $\mathrm{cm}^{-1}$ and $16030 \mathrm{~cm}^{-1}$ in Fig. 1 occur at the opening of the direct channels

$$
\begin{aligned}
& \mathrm{Au}_{2}^{-}\left(v^{\prime \prime}=0\right)+h v \Rightarrow \mathrm{Au}_{2}(v=1)+e^{-}, \\
& \mathrm{Au}_{2}^{-}\left(v^{\prime \prime}=0\right)+h v \Rightarrow \mathrm{Au}_{2}(v=2)+e^{-},
\end{aligned}
$$

respectively, as confirmed by prior measurements using zero electron kinetic energy (ZEKE) spectroscopy ${ }^{11,12}$ in which only electrons of low KE $\left(E_{\text {kin }}<1.5 \mathrm{meV}\right)$ are collected. The surprising aspect of Fig. 1 is the appearance of sharp lines on top of the fairly smooth total electron signal. At this high photon flux used in Fig. 1, the intensity ratios between the smooth signal and the individual lines are strongly perturbed due to saturation effects, but for illustrative purposes, the picture is very useful. The positions and relative intensities of these peaks measured at much lower photon flux $(\sim 1$ $\mu \mathrm{J} / \mathrm{cm}^{2}$ ) are listed in Table I. No other sharp features are observed in the broader range of $16300-15550 \mathrm{~cm}^{-1}$. We attribute the sharp features to resonances in the photodetachment cross section of $\mathrm{Au}_{2}^{-}$created by photoexcitation from the $\mathrm{Au}_{2}^{-}$electronic ground state to different vibrational levels of an autodetaching excited state of the anion.

In order to support this interpretation, several checks were carried out:

(A) In order to ensure that all electrons correspond to detachment from the dimer anion, the following tests were done:

(i) The electron signal decreases by at least two orders of magnitude if the anion beam is switched off. Thus any electrons from background gas, surfaces, the neutral cluster beam, etc. are negligible.

(ii) The detaching laser time delay is changed to a value corresponding to time between the arrival of two anion bunches, i.e., between $\mathrm{Au}^{-}$and $\mathrm{Au}_{2}^{-}$, or $\mathrm{Au}_{2}^{-}$and $\mathrm{Au}_{3}^{-}$. Again the electron signal drops by over two orders of magnitude, suggesting the mass resolution is sufficient to exclude other gold anions. However, the mass resolution is limited and we cannot completely exclude contributions from impurities such as $\mathrm{Au}_{2}\left(\mathrm{H}_{2} \mathrm{O}^{-}{ }^{-}\right.$, or $\mathrm{Au}_{2} \mathrm{O}^{-}$or $\mathrm{Au}_{2} \mathrm{H}_{2}^{--}$. However, in previous measurements on gold cluster cations and anions, the mass spectra appeared very clean with almost no evidence for any mass peaks other than the bare gold clusters. In addition, we did not observe any variation in the detachment spectra even when running the source over several days. It has been our experience with other systems that if metals which can be oxidized are used, the oxide or water impurity peaks can be seen, but they decrease in intensity with time.

(B) The expansion conditions were altered in order to change the temperature of the dimer anions. Figures 2(a) and 2 (b) show what happens to the peaks labeled $C, D, E$, and $F$ measured (a) at normal expansion conditions $\left(T_{\text {vib }} \sim 165 \mathrm{~K}\right)$ and (b) at lower stagnation pressure and thus higher temperature $\left(T_{\text {vib }} \sim 250 \mathrm{~K}\right)$. The vibrational

TABLE I. The positions and relative intensities of the resonances measured at low photon flux $\left(\sim 1 \mu \mathrm{J} / \mathrm{cm}^{2}\right)$ at

\begin{tabular}{|c|c|c|c|c|}
\hline Transition & $\begin{array}{l}\text { Position }^{a} \\
\left(\mathrm{~cm}^{-1}\right)\end{array}$ & $\begin{array}{c}\text { Tentative } \\
\text { assignment }\end{array}$ & $\begin{array}{c}\text { Relative intensity }^{\mathrm{b}} \\
T_{\mathrm{vib}} \sim 165 \mathrm{~K}\end{array}$ & $\begin{array}{c}\text { Relative intensity } \\
T_{\text {vib }} \sim 250 \mathrm{~K}\end{array}$ \\
\hline$A$ & 15738 & $1 \Leftarrow 2$ & 0.02 & 0.03 \\
\hline$B$ & 15757.5 & $0 \Leftarrow 1$ & 0.03 & 0.03 \\
\hline$C$ & 15845 & $3 \Leftarrow 3$ & 0.03 & 0.08 \\
\hline$D$ & 15865 & $2 \Leftarrow 2$ & 0.11 & 0.20 \\
\hline$E$ & 15885.5 & $1 \Leftarrow 1$ & 0.27 & 0.57 \\
\hline$F$ & 15905 & $0 \Leftarrow 0$ & 1 & 1 \\
\hline$G$ & 16012 & $2 \Leftarrow 1$ & 0.08 & 0.12 \\
\hline$H$ & 16033 & $1 \Leftarrow 0$ & 0.14 & 0.15 \\
\hline
\end{tabular}
two different vibrational temperatures.

"Uncertainty $\pm 1 \mathrm{~cm}^{-1}$.

Uncertainty $\pm 30 \%$. 


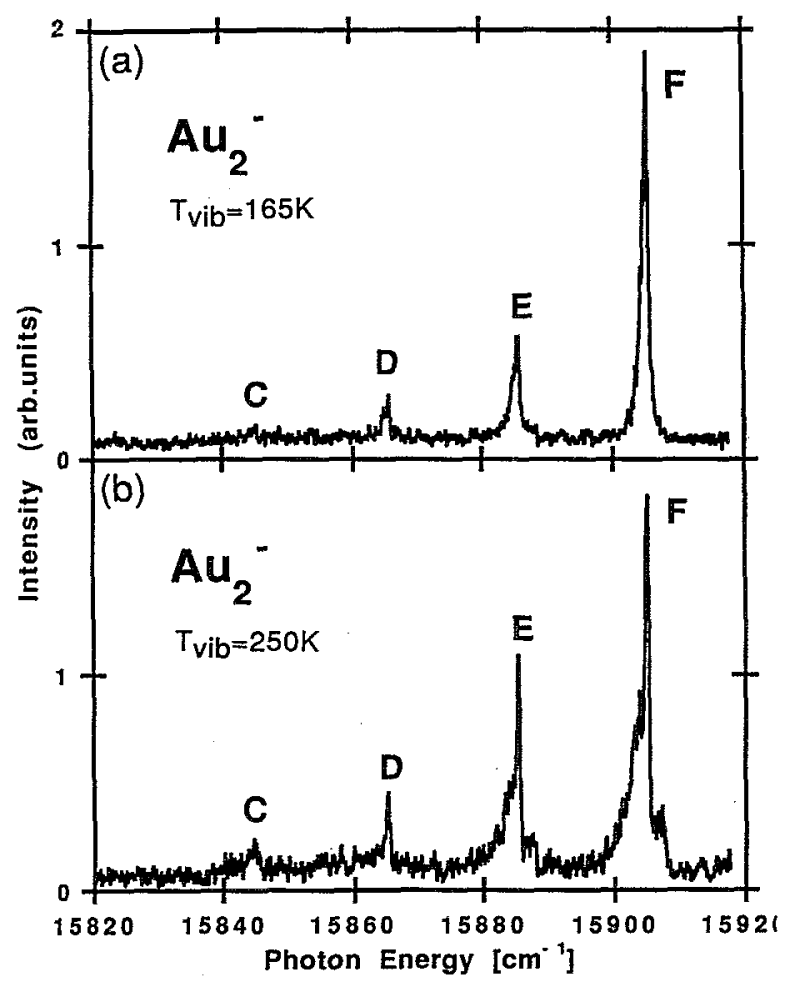

FIG. 2. Expanded view of Fig. 1, taken at two different vibrational temperatures of the anions and a much lower photon flux $\left(\sim 1 \mu \mathrm{J} / \mathrm{cm}^{2}\right)$. The vibrational temperature in (a) is known from other experiments, while the temperature in (b) is calculated with the assumption that the intensities of the peaks reflect the population of the vibrational levels of the electronic ground state of the anion (see the text). At higher temperature (b), the resonances broaden and features $C, D$, and $E$ increase in intensity relative to $F$.

temperature in Fig. 2(a) is known from ZEKE spectroscopy. ${ }^{11}$ The temperature in Fig. 2(b) is estimated with the assumption that $C, D$, and $E$ are hot bands. Generally the small features $A, B, C, D, E$, and $G$ increase in intensity with temperature relative to $F$ and $H$ (see Table I). At the higher temperature, all peaks show a broadening.

\section{DISCUSSION}

The relatively smooth signal which occurs over the entire spectral range is due to direct photodetachment from the gold dimer anion. Assuming an increase in electron signal at each new accessible vibrational transition, the steps at $\sim 15840$ and $\sim 16030 \mathrm{~cm}^{-1}$ are due to the onset of photodetachment from the $\mathrm{Au}_{2}{ }^{-} v^{\prime \prime}=0$ ground state to the $v^{\prime}=1$ and $v^{\prime}=2$ vibrational states of the neutral dimer, respectively [reactions (3) and (4)]. In a previous study ${ }^{11}$ using zero electron kinetic energy spectroscopy, the thresholds of the $1 \Leftarrow 0$ and $2 \Leftarrow 0$ transitions were measured to be $15831 \pm 4$ and $16017 \pm 4 \mathrm{~cm}^{-1}$, respectively, in agreement with the position of the observed steps.

The strongest hint that the observed resonances might be due to transitions into an autoionizing excited state of the dimer anion is the observation that certain spacings between the resonances coincide with the vibrational spacing of the dimer anion ground state $\left(149 \mathrm{~cm}^{-1}\right) .^{11}$ These are, for instance, the frequencies between the resonances labeled $F$ and
$B$, and between $E$ and $H$. If one assumes the two strongest features $(F$ and $H)$ are transitions from $\mathrm{Au}_{2}^{-}\left(v^{\prime \prime}=0\right)$ to two different vibrational levels of the excited state, the vibrational frequency of the excited state is $128 \pm 2 \mathrm{~cm}^{-1}$. The less intense features $B, E$, and $G$ can be assigned as transitions from the $v^{\prime \prime}=1$ level of the anion ground state shifted by $21 \mathrm{~cm}^{-1}\left(149-128 \mathrm{~cm}^{-1}\right)$ relative to the cold bands. The features $A, C$, and $D$ are at positions, where peaks corresponding to transitions from higher excited vibrational levels of the anion electronic ground state are expected. Indeed, as expected, and as shown in Fig. 2 and Table I, the relative intensities of these features $(A, B, C, D, E, G)$ increase with increasing temperature of the anions.

The observation of this relative low number of features $(A-H)$ in a narrow spectral range can be explained by assuming that the feature $F$ is the $0 \Leftarrow 0$ transition and $H$ is the $1 \Leftarrow 0$ transition. The absence of a peak at $15786 \mathrm{~cm}^{-1}$ (15 914-128 $\mathrm{cm}^{-1}$ ) is thus easily explained. $F$ is then the lowest possible transition of the $v^{\prime} \Leftarrow 0$ series and $B$ is the lower limit of the $v^{\prime} \Leftarrow 1$ series, in agreement with the observations. Alternatively, the absence of the feature at 15786 $\mathrm{cm}^{-1}$ could mean that the corresponding vibrational level is not able to decay via electron emission, but it is not very likely, because the decay by electron emission is energetically allowed (i.e., excitation is above the detachment threshold).

If $F$ is the $0 \Leftarrow 0, H$ the $1 \Leftarrow 0$, and $B$ the $0 \Leftarrow 1$ transition, one expects additional features at higher energies $(>16100$ $\mathrm{cm}^{-1}$ ). The absence of features at higher energies can be explained, if one interprets intensity distribution of the resonances as a Franck-Condon pattern of a transition between electronic states with similar shapes of the potential wells and equilibrium distances. Then the Franck-Condon factor is about a factor of 7 lower for $F$ than $H$. If a similar drop occurs between the $1 \Leftarrow 0$ and $2 \Leftarrow 0$ transitions, the next transition $(2 \Leftarrow 0)$ is likely too weak to be seen, thus accounting for its absence. However, we cannot rule out that the absence of transitions into higher vibrational levels could be due to the fact that some other decay channel such as predissociation opens up and effectively competes with a long-lived autodetaching state.

According to the prior discussion, the peaks $F, E, D$, and $C$ thus correspond to the transitions $0 \Leftarrow 0,1 \Leftarrow 1,2 \Leftarrow 2$, and $3 \Leftarrow 3$, respectively. If the equilibrium distances of the two involved electronic states are similar, the Franck-Condon factors of each of these lines should be similar and close to 1 . Thus, their relative intensities should reflect the population of the vibrational levels of the electronic ground state of the anions. The temperature at normal expansion conditions is known to be $165 \pm 30 \mathrm{~K},{ }^{11}$ which should result in a decrease of the population going from one level to the next higher by a factor of $3.7\left(\Delta E=149 \mathrm{~cm}^{-1}\right)$. This is in good agreement with the observations [Fig. 2(a) and Table I]. The relative intensities displayed in Fig. 2(b) correspond to a vibrational temperature of $\sim 250 \mathrm{~K}$. In addition, the resonances show a broadening, which can be explained by rotational effects.

The observed resonances can be understood as transitions into an excited state of the anion $15905 \mathrm{~cm}^{-1}(0 \Leftarrow 0)$ above the anion ground state, but within $\sim 250 \mathrm{~cm}^{-1}$ above 
the threshold for production of the neutral ground state. It has a vibrational frequency of $128 \pm 2 \mathrm{~cm}^{-1}$ and an equilibrium distance similar to the ground state of the anion. In the following, we will discuss possible excited states of $\mathrm{Au}_{2}^{-}$ within a simple orbital model.

Excited anionic states of atoms and molecules are well known as resonances in electron scattering experiments on neutrals $^{4,5}$ [see reaction (1)]. There are two different types-shape resonances and Feshbach resonances. For shape resonances, the decay channel by autodetachment into the neutral parent state (ground or excited state) is energetically allowed. The electron is weakly bound in the potential of the neutral core by an angular momentum barrier. The decay occurs by tunneling of the electron through the barrier. These resonances lie above the parent state and the electron must be bound in an orbital with nonzero angular momentum. Feshbach resonances are energetically located below the parent state, i.e., the parent state must be an excited state of the neutral. A Feshbach resonance of the neutral ground state would be simply a stable negative ion. The electron is bound in the potential of the neutral with a positive electron affinity and will decay via electronic autodetachment, i.e., the neutral core relaxes into its ground state transferring the energy to the additional electron which is ejected.

Recently two other types of weakly bound metastable excited states of anions were studied by a photodetachment spectroscopy. In one case, the electron is bound by a permanent dipole moment of a molecule. ${ }^{8,9}$ The decay occurs by vibrational autodetachment, i.e., the energy necessary for the electron ejection is extracted from the vibrational excitation of the neutral core. Resonances found approximately 50 $\mathrm{meV}$ above the photodetachment threshold of $\mathrm{Au}_{6}{ }^{-}$are interpreted as vibrational autodetachment from a surface state of this very small metal particle. ${ }^{10}$ In other words, the electron is bound by image charges of the metal cluster. In both cases, the electron is very weakly bound and thus its orbital is very extended. The vibrational frequencies of the negative ion are expected to be similar to those of the neutral molecule.

It is not likely, that the excited state of $\mathrm{Au}_{2}^{-}$is one of the weakly bound types for two reasons:

(a) the vibrational frequency $\left(128 \mathrm{~cm}^{-1}\right)$ is lower than that of the neutral ground state $\left(190.9 \mathrm{~cm}^{-1}\right)^{13}$ and even lower than that of the anion ground state $\left(149 \mathrm{~cm}^{-1}\right) ;^{11}$

(b) $\mathrm{Au}_{2}{ }^{-}$has no permanent dipole moment and is so small that it seems unlikely that the electron binding can be explained by classical electrostatic phenomena.

Instead we propose that there exists an electronic excited state of the dimer anion, which decays by electronic autodetachment similar to Feshbach or shape resonances. The valence states of $\mathrm{Au}_{2}^{-}$have a relatively simple structure consisting of a filled bonding $\sigma$ orbital and a half-filled nonbonding $\sigma^{*}$ orbital together with the two closed $d$ shells. The three lowest excited states of $\mathrm{Au}_{2}^{-}$according to a simple molecular orbital picture are:

(A) Excitation of one of the bonding electrons from the filled $\sigma$ orbital into the half-filled $\sigma^{*}$ orbital. This transition will be dipole allowed if the $\sigma^{*}$ orbital has some contribution from $p$ orbitals (hybridization). This would also explain the relatively high electron affinity and the nonbonding rather than antibonding character of the $\sigma^{*}$ orbital. ${ }^{13}$ This state is a Feshbach resonance, because it is energetically below its parent state which is the corresponding single electron $\sigma \Rightarrow \sigma^{*}$ excitation of the neutral. It can only decay into the ground state of the neutral by a two electron process. The removal of a bonding $\sigma$ electron should weaken the bonding which is usually accompanied by a decrease of the vibrational frequencies $\left(<149 \mathrm{~cm}^{-1}\right)$. The $\sigma-\sigma^{*}$ spacing for $\mathrm{Cu}_{2}^{-}$and $\mathrm{Ag}_{2}{ }^{-}$is about $2 \mathrm{eV}^{14}$ and is expected to be similar for $\mathrm{Au}_{2}^{-}$.

(B) Excitation of a $d$ electron into the $\sigma^{*}$ orbital ( $d-s$ promotion). This transition is again dipole allowed in the case of $s-p$ hybridization. From photodetachment data, ${ }^{14}$ the $d-s$ promotion energy of $\mathrm{Cu}_{n}^{-}$is estimated to be about 2 $\mathrm{eV}$. From a comparison with neutral atoms, ${ }^{15}$ the $d-s$ promotion energy of $\mathrm{Au}$ ( $J$ averaged) is similar to that of $\mathrm{Cu}$ due primarily to relativistic effects. The decay would be similar to case.(A).

(C) Transition of the single electron in the nonbonding $\sigma^{*}$ orbital into a higher state, e.g., the $\pi_{u}$-bonding orbital. the remaining core would be expected to have nearly the same symmetry as the neutral ground state. Such a state would be a shape resonance with the ground state of the neutral as the parent state. The vibrational frequencies would be expected to be similar to the neutral ground state. This behavior is found in calculations ${ }^{16}$ for the ${ }^{2} \Pi_{u}$ excited state of $\mathrm{Cu}_{2}^{-}$. This type of excited state would be accompanied by an increase of the vibrational frequencies compared to ground state $\mathrm{Au}_{2}^{-}$and appears unlikely to be the correct interpretation of the observed features.

From the above consideration, the excited state is probably either a $\sigma \Rightarrow \sigma^{*}$ excitation or a $d-s$ promotion accompanied by a decrease of the vibrational frequency. Using the terminology of electron scattering, we suggest the state is a Feshbach resonance decaying via electronic autodetachment. This is of course only a tentative assignment and elaborate calculations are likely to be necessary to obtain a better understanding of the data.

\section{CONCLUSION}

Resonances in the photodetachement cross section of $\mathrm{Au}_{2}{ }^{-}$are explained as photoexcitation of $\mathrm{Au}_{2}^{-}$into an autodetaching excited state. The comparison of data taken at different vibrational temperatures allows a tentative assignment of the vibrational transitions. The unexpected low vibrational frequency excludes the interpretation of the excited state as an image charge bound state as proposed for $\mathrm{Au}_{6}{ }_{6}^{-}$, i.e., a weakly bound state of the additional electron with a neutral dimer in its ground state as a core. We propose that the resonances are due to electronic autodetachment from an electronic excited state of the anion, where either an electron from the $d$ orbital or from the bonding $\sigma$ orbital is excited into the nonbonding $\sigma^{*}$ orbital. In both case, a vibrational frequency lower than that of the ground state neutral or anion is expected and the excitation energy would be about $2 \mathrm{eV}$. This state is entirely analogous to a Feshbach resonance in electron scattering experiments, decaying by clectronic autodetachment. 
'(a) D. G. Leopold, J. H. Ho, and W. C. Lineberger, J. Chem. Phys. 86, 1715 (1987). (b) U. Hefter, R. D. Mead, P. A. Schulz, and W. C. Lineberger, Phys. Rev. A 28, 1429 (1983).

${ }^{2}$ O. Cheshnovsky, S. H. Yang, C. L. Pettiette, M. J. Craycraft, and R. E. Smalley, Rev. Sci. Instrum, 58, 2131 (1987).

${ }^{3}$ G. Gantefoer, K. H. Meiwes-Broer, and H. O. Lutz, Phys. Rev. A 37, 2716 (1988).

${ }^{4}$ G. J. Schulz, Rev. Mod.Phys. 45, 378 (1973); 45, 423 (1973).

${ }^{5}$ M. Allan, J. Electron. Spectrosc. Relat. Phenom. 48, 219 (1989).

${ }^{6}$ V. E. Bondebey and J. H. English, J. Chem.Phys. 74, 6978 (1981).

'See, e.g., J. Berkowitz, Photoabsorption, Photoionization, and Photoelectron Spectroscopy (Academic, New York, 1979).

${ }^{8}$ R. L. Jackson, A. H. Zimmerman, and J. I. Brauman, J. Chem. Phys. 71, 2088 (1979).

'J. Marks, J. I. Brauman, R. D. Mead, K. R. Lykke, and W. C. Lineberger, J. Chem. Phys. 88, 6785 (1988).
${ }^{10} \mathrm{~K}$. J. Taylor, C. Jin, J. Conceicao, Lai-Sheng Wang, O. Cheshnovsky, B. R. Johnson, P. J. Nordlander, and R. E. Smalley J. Chem. Phys. (submitted).

${ }^{11}$ G. F. Gantefoer, D. M. Cox, and A. Kaldor, J. Chem. Phys. (in press). ${ }^{12}$ Note that in ZEKE spectroscopy, electron signals are only observed in a narrow ( $1.5 \mathrm{meV}$ ) energy region around the threshold energy for each vibronic transition.

${ }^{13}$ J. Hoe, K. M. Ervin, and W. C. Lineberger, J. Chem. Phys. (in press).

${ }^{14}$ (a) O. Cheshnovsky, K. J. Taylor, J. Conceicao, and R. E. Smalley, Phys. Rev. Lett. 64, 1785 (1990); (b) G. Gantefoer and K. H. Meiwes-Broer (unpublished data).

${ }^{15}$ C. E. Moore, Atomic Energy Levels (Nat. Bur. Stand. NSRDS-NBS 35, U.S. GPO, Washington, D.C., 1971), Vols. I-III.

${ }^{16} \mathrm{C}$. W. Bauschlicher, Jr., S. R. Langhoff, and P. R. Taylor, J. Chem. Phys. 88, 1041 (1988). 